\title{
Intraoperative MRI-based elastic fusion for anatomically accurate tractography of the corticospinal tract: correlation with intraoperative neuromonitoring and clinical status
}

\author{
Sebastian Ille, MD, ${ }^{1,2}$ Axel Schroeder, ${ }^{1,2}$ Arthur Wagner, MD,,2 Chiara Negwer, MD,1 \\ Kornelia Kreiser, MD, ${ }^{3}$ Bernhard Meyer, MD, ${ }^{1}$ and Sandro M. Krieg, MD, MBA ${ }^{1,2}$
}

${ }^{1}$ Department of Neurosurgery, ${ }^{2}$ TUM Neuroimaging Center, and ${ }^{3}$ Department of Diagnostic and Interventional Neuroradiology, Technical University of Munich, School of Medicine, Klinikum rechts der Isar, Munich, Germany

\begin{abstract}
OBJECTIVE Tractography is a useful technique that is standardly applied to visualize subcortical pathways. However, brain shift hampers tractography use during the course of surgery. While intraoperative MRI (ioMRI) has been shown to be beneficial for use in oncology, intraoperative tractography can rarely be performed due to scanner, protocol, or head clamp limitations. Elastic fusion (EF), however, enables adjustment for brain shift of preoperative imaging and even tractography based on intraoperative images. The authors tested the hypothesis that adjustment of tractography by ioMRI-based EF (IBEF) correlates with the results of intraoperative neuromonitoring (IONM) and clinical outcome and is therefore a reliable method.

METHODS In 304 consecutive patients treated between June 2018 and March 2020, 8 patients, who made up the basic study cohort, showed an intraoperative loss of motor evoked potentials (MEPs) during motor-eloquent glioma resection for a subcortical lesion within the corticospinal tract (CST) as shown by ioMRI. The authors preoperatively visualized the CST using tractography. Also, IBEFs of pre- and intraoperative images were obtained and the location of the CST was compared in relation to a subcortical lesion. In 11 patients (8 patients with intraoperative loss of MEPs, one of whom also showed loss of MEPs on IBEF evaluation, plus 3 additional patients with loss of MEPs on IBEF evaluation), the authors examined the location of the CST by direct subcortical stimulation (DSCS). The authors defined the IONM results and the functional outcome data as ground truth for analysis.
\end{abstract}

RESULTS The maximum mean \pm SD correction was $8.8 \pm 2.9$ (range 3.8-12.0) $\mathrm{mm}$ for the whole brain and $5.3 \pm 2.4$ (range 1.2-8.7) $\mathrm{mm}$ for the CST. The CST was located within the lesion before IBEF in 3 cases and after IBEF in all cases $(p=0.0256)$. All patients with intraoperative loss of MEPs suffered from surgery-related permanent motor deficits. By approximation, the location of the CST after IBEF could be verified by DSCS in 4 cases.

CONCLUSIONS The present study shows that tractography after IBEF accurately correlates with IONM and patient outcomes and thus demonstrates reliability in this initial study.

https://thejns.org/doi/abs/10.3171/2020.10.FOCUS20774

KEYWORDS glioma; intraoperative MRI; intraoperative neuromonitoring; paresis; tractography

$\mathrm{T}$ HE microsurgical resection of motor-eloquent gliomas requires avoidance of surgery-related motor deficits while achieving a maximum extent of resection (EOR) for optimal oncological treatment. ${ }^{1-4}$ The gold standard technique for the surveillance of motor functions is intraoperative neuromonitoring (IONM), and the reliability and validity of this method are commonly accepted..$^{5-9}$
Additionally, tractography offers the opportunity to visualize the corticospinal tract (CST) and is regularly used by many neurosurgeons to get a better idea of subcortical anatomy and its tumor-induced displacement. Several studies comparing the algorithm-based approach of tractography to the gold standard technique of intraoperative direct subcortical stimulation (DSCS) showed good con-

ABBREVIATIONS CST = corticospinal tract; $D C=$ distortion correction; $D C S=$ direct cortical stimulation; $D S C S=$ direct subcortical stimulation; $D T I=$ diffusion tensor imaging; DWI = diffusion-weighted imaging; EF = elastic fusion; EOR = extent of resection; FT = fiber tracking; GTR = gross-total resection; IBEF = ioMRI-based EF; ioMRI = intraoperative MRI; IONM = intraoperative neuromonitoring; MEP = motor evoked potential; $n$ TMS = navigated transcranial magnetic stimulation; ROI = region of interest; STR = subtotal resection; TES = transcranial electrical stimulation; T1 + CA = T1-weighted sequence with contrast administration.

SUBMITTED August 29, 2020. ACCEPTED October 26, 2020.

INCLUDE WHEN CITING DOI: 10.3171/2020.10.FOCUS20774. 
cordance between the methods..$^{10-15}$ With the aim of validating the reliability of fiber tracking (FT), multiple studies measured distances between DSCS points and the CST as visualized by tractography. ${ }^{13,14,16-23}$ The findings of these studies led to the rule of thumb that $1 \mathrm{~mA}$ corresponds to $1 \mathrm{~mm} .{ }^{21,24}$ Surgeons continue to question the intraoperative use of tractography mainly because of its inaccuracy after partial resection and the resulting brain shift, which is further aggravated toward the end of surgery when the resection reaches highly eloquent tracts, and tractography could be most useful if it was reliable.

Meanwhile, high-volume centers for glioma surgery standardly use the additional technique of intraoperative MRI (ioMRI). The benefit of using ioMRI to maximize EOR, overall survival, and patient quality of life has already been shown. ${ }^{25-27}$ In combination with neuronavigation, ioMRI offers the opportunity to image the brain with an open dura mater and thereby visualize the extent of brain shift.

However, although IONM is still useful after ioMRI, ${ }^{28-31}$ results of preoperative tractography cannot simply be fused with the ioMRI scan due to major inaccuracies based on the missing 3D deformation of the tractography and the correction for brain shift. Furthermore, a new tractography method based on ioMRI scans is limited by the scanner, head clamp, or time limitations.

With this in mind, prior retrospective methodological studies have already proven the feasibility of elastic fusion (EF) for the reduction of geometrical image distortions of simultaneously scanned MRI sequences by distortion correction (DC). ${ }^{32}$ Based on these algorithms, MRI sequences that have been scanned at different time points such as pre- and intraoperatively can also be fused to adjust for anatomical changes like tumor resection or brain shift after opening of the dura. Most importantly, this ioMRIbased EF (IBEF) allows for adjustment of preoperative tractography. However, the accuracy and reliability of these algorithms as well as the relevance of tractography must still be evaluated by clinical parameters.

Hence, the basis of the present study is the hypothesis that the fusion of pre- and intraoperative MRI scans by IBEF correlates better with clinical outcome than preoperative diffusion tensor imaging (DTI) without IBEF. Therefore, adjustment of the CST as visualized by tractography with and without IBEF was correlated with clinical outcome and IONM in terms of continuous motor evoked potential (MEP) monitoring and distances to the CST via DSCS.

\section{Methods \\ Ethics}

This study was approved by the local ethics board. We performed the study in accordance with the Declaration of Helsinki. All included patients provided written informed consent.

\section{Study Protocol}

We implemented the present study protocol with the aim of correlating the results of pre- and intraoperative neurophysiology and imaging in terms of function-based
DTI FT, IONM, and the patients' functional outcome. Therefore, we prospectively included patients with brain tumors as defined by preoperative MRI scan scheduled for microsurgical resection. We excluded patients younger than 18 years of age or with general MRI exclusion criteria such as cochlear implants or pacemakers. The study was performed at the Department of Neurosurgery, Klinikum rechts der Isar, Technische Universität München, Germany.

We used navigated transcranial magnetic stimulation (nTMS) to identify MEP-positive cortical areas for the exact seeding of function-based DTI FT. Patients then underwent microsurgical tumor resection using neuronavigation, continuous transcranial electrical stimulation (TES), or direct cortical stimulation (DCS) in all cases. Additionally, we performed subcortical mappings by DSCS in appropriate cases. When maximum tumor resection was completed, we transferred the patient to the ioMRI scanner. Based on these new images, we then performed IBEF. After the ioMRI scan, either the resection was continued or wound closure was performed. Postoperatively, patients were examined on day 5 and at a 3-month follow-up.

\section{DTI FT of the CST}

Preoperatively, all patients underwent a structural MRI scan (3T MRI scanner, Achieva, Philips Medical Systems) according to the standard MRI protocol, including a 3D FLAIR sequence, a 3D gradient echo sequence with intravenous contrast administration (T1-weighted sequence with contrast administration [T1 + CA] ), and DTI sequences with 32 orthogonal sequences. As a standard procedure, we set the regions of interest (ROIs) for DTI FT by positive MEP cortical sites as determined by preoperative nTMS mapping, and we set a second ROI within the ipsilateral brainstem. ${ }^{33}$ Hence, we combined neurophysiology and tractography in terms of an MEP-based DTI FT to ensure the visualization of fibers originating from true motoreloquent regions. For tractography, we used a standard deterministic algorithm with fiber assignment by continuous tracking (FACT; iPlanNet Cranial 3.0.1, Brainlab AG). Based on earlier publications, ${ }^{34}$ we used a fractional anisotropy of $0.1-0.2$ and minimal fiber length of $70-110 \mathrm{~mm}$ to generate highly reproducible tractography results.

\section{Intraoperative Neuromonitoring}

For TES, we used an ISIS neurostimulator and subcutaneous stimulation needles (inomed Medizintechnik) at C3 and C4 based on the 20-10 EEG system. For DCS, we used a strip electrode with 4 contacts over the motor cortex and an Fpz needle electrode for the cathodic pole (inomed Medizintechnik). We recorded and documented compound muscle action potentials within the abductor pollicis brevis muscle, adductor digiti minimi muscle, biceps brachii muscle, and tibialis anterior muscle. During tumor resection, we measured MEPs continuously. We considered amplitude losses or declines of $>50 \%$ from baseline significant in the case of a missing recovery above $50 \%$ baseline. For TES, we documented baseline measurements after opening of the dura. We ruled out technical issues and anesthesiological causes of decline before informing the surgeon. For DSCS, we used a monopolar 


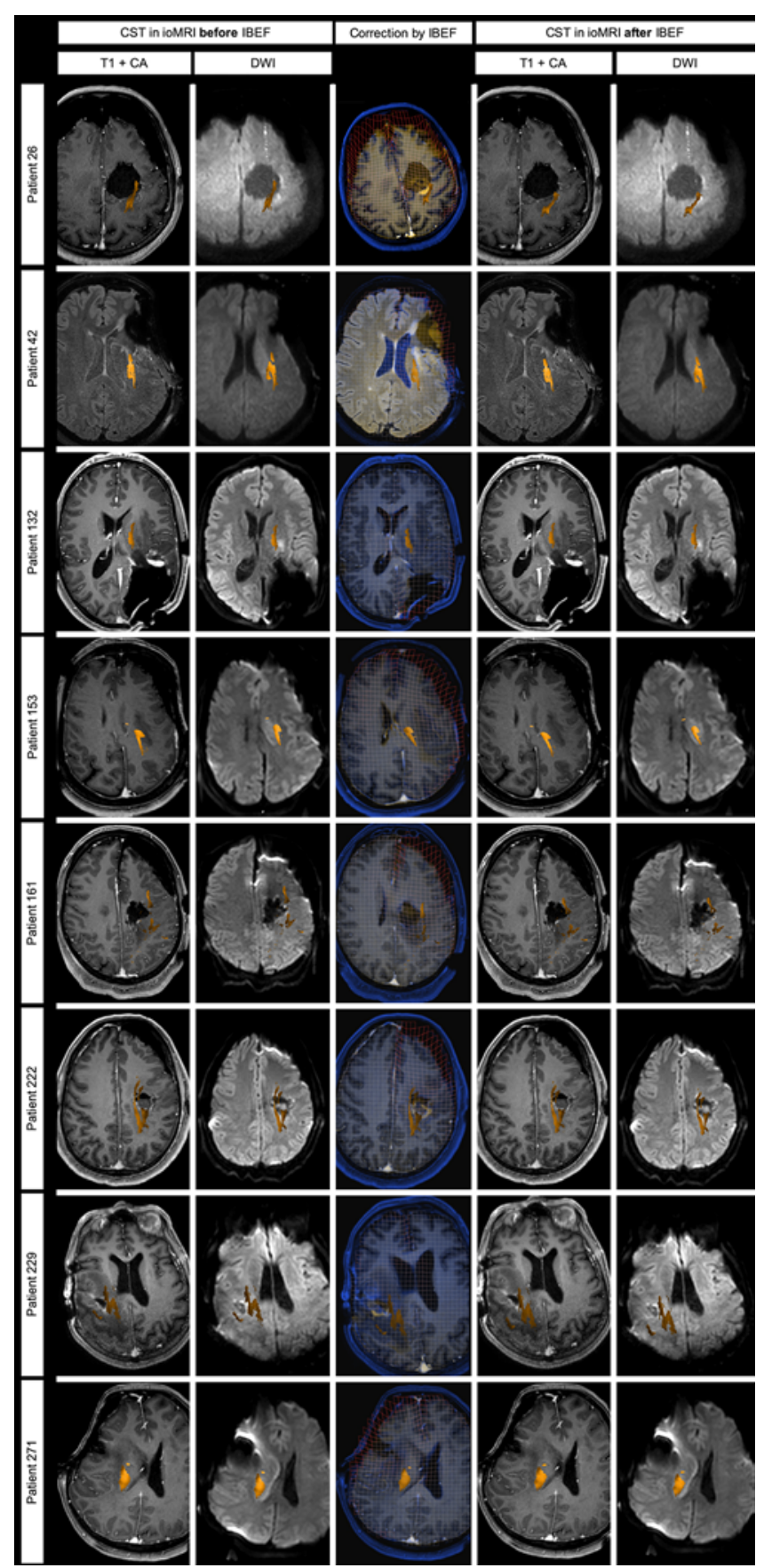

FIG. 1. Correction by IBEF. The figure shows the CST before correction by IBEF within the ioMRI T1 + CA (column 1) and DWI (column 2), as well as the correction of the CST by IBEF within the ioMRI T1 + CA (column 4) and DWI (column 5). Column 3 shows the algorithm-based correction that was calculated through the EF of the preoperative (yellow) and intraoperative (blue) MRI. Red areas indicate the extent of deformation and correction.

handheld probe (inomed Medizintechnik) with a cathodal stimulation and possible intensities between 3 and $30 \mathrm{~mA}$. Stimulation points with the lowest current intensity which still elicited an MEP response were saved by neuronavigation and microscope screenshots.
TABLE 1. Patient, tumor, and surgery characteristics

\begin{tabular}{|c|c|}
\hline & Value \\
\hline No. of patients & 11 \\
\hline Age in yrs & $53.5 \pm 16.1(25-75)$ \\
\hline \multicolumn{2}{|l|}{ Tumor type (WHO grade) } \\
\hline Oligodendroglioma (II) & 1 \\
\hline Oligodendroglioma (III) & 1 \\
\hline Astrocytoma (III) & 1 \\
\hline Glioblastoma & 8 \\
\hline \multicolumn{2}{|l|}{ Recurrence } \\
\hline Yes & 5 \\
\hline No & 6 \\
\hline \multicolumn{2}{|l|}{ Hemisphere } \\
\hline Rt & 5 \\
\hline Lt & 6 \\
\hline \multicolumn{2}{|l|}{ Location } \\
\hline Precentral & 5 \\
\hline Insular & 5 \\
\hline Temporal & 1 \\
\hline Duration of op in mins & $264.9 \pm 60.1(159-391)$ \\
\hline Duration of ioMRI in mins* & $65.0 \pm 7.1(50-70)$ \\
\hline \multicolumn{2}{|l|}{ Initial EOR } \\
\hline GTR & 8 \\
\hline STR & 3 \\
\hline Resection after ioMRI & 1 \\
\hline \multicolumn{2}{|l|}{ Final EOR } \\
\hline GTR & 9 \\
\hline STR & 2 \\
\hline
\end{tabular}

Values are presented as the number of patients or the mean $\pm \mathrm{SD}$ (range).

* The ioMRI duration was documented as the time between insertion and removal of the collagen sponge.

\section{Intraoperative MRI Scan}

For ioMRI scans, we used a 2-room ioMRI setup (3T MR scanner, Ingenia, Philips Medical Systems) and an MRI-compatible head clamp including an 8-channel coil array (Noras MRI products) for all glioma cases. After completing the initial resection and hemostasis, we refilled the resection cavity with Ringer's solution and closed it with a collagen sponge and 2-stitch suture. Afterward, we covered the approach with multiple sterile layers. We removed the IONM needles for safety and image-quality reasons and transferred the patient to the ioMRI scanner when checklists were completed..$^{28,29,35}$

\section{IBEF Procedure}

IBEF was performed according to the following procedure. First, segmentation of the preoperative MRI scan is performed. During the ioMRI scan, the anatomical 3D T1 sequence is used for fusion of preoperative and new intraoperative images. Afterward, the IBEF algorithm calculates the deformation based on the comparison of pre- and intraoperative images, finite element analysis, and gravity. Within this step, preoperatively determined objects such 
Ille et al.

TABLE 2. IONM and functional outcome

\begin{tabular}{rccccccc}
\hline \multirow{2}{*}{$\begin{array}{c}\text { Patient } \\
\text { No. }\end{array}$} & \multicolumn{2}{c}{ IONM } & & \multicolumn{4}{c}{ Motor Status } \\
\cline { 2 - 3 } \cline { 6 - 7 } \cline { 6 - 7 } 26 & Technique & Loss & & Preop & Postop & 3 Mos Postop \\
\hline 42 & TES & DCS & $X$ & & 4 & 0 & 0 \\
\hline 132 & TES & $X$ & & 5 & 0 & 0 \\
\hline 153 & TES & $X$ & 4 & 1 & 1 \\
\hline 161 & TES & $X$ & 4 & 0 & 0 \\
\hline 222 & DCS & $X$ & 4 & 1 & 1 \\
\hline 229 & TES + DSCS & Decline & 4 & 0 & 0 \\
\hline 271 & TES & $X$ & 5 & 0 & 0 \\
\hline 255 & TES + DSCS & - & 5 & 5 & 5 \\
\hline 273 & TES + DSCS & - & 5 & 5 & 5 \\
\hline 298 & TES + DSCS & - & 2 & 2 & 2 \\
\hline
\end{tabular}

$-=$ no intraoperative loss of MEPs; $X=$ intraoperative loss of MEPs. Results of IONM by TES, DCS, and DSCS and the motor status of patients measured by the British Medical Research Council scale preoperatively, postoperatively, and during the 3-month follow-up. Decline was documented in case of amplitude decrease $>50 \%$ compared to baseline measurement without recovery.

as the CST are adjusted to new conditions based on the ioMRI scan. When the calculation is completed, the EF result must be verified and accepted by the surgeon. Finally, the IBEF result and the ioMRI can then be used for further resection (Brainlab Elements, Brainlab AG).

\section{Data Analysis}

For the determination of EOR, we used a threshold of $5 \%$ of a residual tumor to differentiate between gross-total resection (GTR) and subtotal resection (STR).,36 We per- formed clinical examinations including documentation of the motor status in accordance with the British Medical Research Council scale preoperatively, postoperatively, and at the 3-month follow-up. The latter defined the patients' long-term motor outcome. We measured maximum deformations for the whole brain and the CST as a mean value of image correction via IBEF. For the present analysis, we evaluated the localization of the noncorrected CST (before the application of IBEF) and the corrected CST (after the application of IBEF) within clinically relevant ioMRI sequences. Therefore, we chose sequences that are essential for the evaluation of a residual tumor and tissue resection (FLAIR for low-grade gliomas; T1 + CA for high-grade gliomas) and diffusion-weighted imaging (DWI) for the detection of subcortical ischemia (Fig. 1). We measured the mean total correction of the CST within the plane of the lesion (ischemia or resection) of the CST. Also, we measured the maximum correction of preoperative imaging by IBEF within the same plane. To confirm the results of CST correction by IBEF, we additionally measured distances between the CST and subcortical stimulation points. All values are presented as the mean \pm standard deviation.

We used GraphPad Prism 8 software to perform statistical calculations. A p value $<0.05$ was considered significant. To calculate the difference between correlations before and after the application of IBEF, we used Fisher's exact test.

\section{Results}

\section{Patient Characteristics and Surgical Details}

Between June 2018 and March 2020, we screened 304 patients who underwent microsurgical glioma resection. In 8 of these patients $(2.6 \%, 4$ females), we documented an intraoperative loss or decline of MEPs during the resection of motor-eloquent gliomas. We examined parts of this

TABLE 3. Correction of the CST by IBEF

\begin{tabular}{|c|c|c|c|c|c|c|c|c|}
\hline \multirow{2}{*}{$\begin{array}{c}\text { Patient } \\
\text { No. }\end{array}$} & \multicolumn{2}{|c|}{ Correction of CST } & \multicolumn{2}{|c|}{ Maximum Correction } & \multicolumn{2}{|c|}{ CST in Ischemia } & \multicolumn{2}{|c|}{ Correlation After IBEF } \\
\hline & In Plane* & Total† & In Plane & Total & Before IBEF & After IBEF & IONM & Outcome \\
\hline 26 & 8.3 & 8.7 & 11.6 & 12.0 & - & $x$ & $x$ & $x$ \\
\hline 42 & 5.2 & 5.6 & 8.1 & 8.6 & - & $x$ & $x$ & $x$ \\
\hline 132 & 2.0 & 2.4 & 5.1 & 5.2 & - & $x$ & $x$ & $x$ \\
\hline 153 & 4.9 & 5.7 & 11.0 & 11.9 & Partial & $X$ & $X$ & $X$ \\
\hline 161 & 7.5 & 8.2 & 9.1 & 9.5 & - & $x$ & $x$ & $x$ \\
\hline 222 & 5.0 & 5.2 & 8.0 & 8.2 & $x$ & $x$ & $X$ & $x$ \\
\hline 229 & 2.1 & 3.2 & 3.7 & 3.8 & - & $x$ & $x$ & $x$ \\
\hline 271 & 3.7 & 4.1 & 9.2 & 9.3 & Partial & $x$ & $x$ & $x$ \\
\hline 255 & 7.6 & 7.7 & 11.4 & 11.5 & - & - & $x$ & $X$ \\
\hline 273 & 0.9 & 1.2 & 8.9 & 10.1 & - & - & $X$ & $X$ \\
\hline 298 & 7.9 & 8.0 & 11.1 & 11.4 & - & - & $x$ & $x$ \\
\hline \multicolumn{9}{|c|}{$\begin{array}{l}-=\text { no; } X=\text { yes. } \\
\text { Measurements of CST correction and maximum correction of the whole brain after the application of IBEF and cor- } \\
\text { relation of CST location after correction by IBEF with the results of IONM and the functional outcome of patients as } \\
\text { described in Table } 2 \text {. } \\
\text { * "In plane" measurements describe the correction within the plane shown in Fig. } 1 . \\
\dagger \text { Total measurements describe the maximum correction at the point of the "in plane" measurement. }\end{array}$} \\
\hline
\end{tabular}


cohort in another subanalysis for the time point of detection of subcortical ischemia by ioMRI (S. Ille, S. Schoen, B. Wiestler, B. Meyer, and S. M. Krieg, unpublished data, April 2020).

Table 1 summarizes patient and tumor characteristics and information on the course of surgeries. Table 2 shows detailed information on IONM results and the patients' clinical outcome for the 11 patients included in the final analyses (8 patients with an intraoperative loss of MEPs, one of whom underwent additional evaluation of IBEF results by DSCS, plus 3 additional patients with no intraoperative loss of MEPs).

\section{Adjustment of the CST by IBEF}

The maximum correction of preoperative FLAIR and T1 + CA imaging by IBEF was $8.8 \pm 2.9$ (range 3.8-12.0) $\mathrm{mm}$ in total and $8.4 \pm 2.7$ (range $3.7-11.6$ ) $\mathrm{mm}$ within the plane of the lesion (ischemia or resection) within the CST or the plane of the DSCS point. The mean total corrections of the CST were $5.3 \pm 2.4$ (range $1.2-8.7$ ) $\mathrm{mm}$ and $4.8 \pm 2.5$ (range $0.9-8.3$ ) $\mathrm{mm}$ within the plane of the lesion detection of the CST or the plane of the DSCS point. The CST was located within the region of ischemia or resection before the adjustment by IBEF in 3 cases $(37.5 \%)$ and after the adjustment by IBEF in all cases with an intraoperative loss of MEPs. Using the localization of the CST within the lesioned region as well as IONM and clinical outcome as the ground truth for comparison purposes, the relocation of the CST by IBEF was statistically significantly different $(\mathrm{p}=0.0256)$ for patients with an intraoperative loss of MEPs. In all cases, we confirmed the direction of the CST's correction to be as suspected according to the expected brain shift based on tumor mass reduction and the patient's positioning. Table 3 and Fig. 1 show detailed information on the correction by IBEF.

\section{Verification by DSCS}

In addition to the 8 patients who showed an intraoperative loss of MEPs, DSCS measurements were performed in 3 additional patients without an intraoperative loss of MEPs and in 1 patient of the initial cohort (patient 229). Characteristics of these patients are included in Tables 1-3. Figures 2 and 3 show the procedures of saving DSCS points and measuring distances between the CST and DSCS points. DSCS intensities were $8.8 \pm 3.8$ (range 5-13) $\mathrm{mA}$ and distances were $8.9 \pm 5.1$ (range 3.6-14.5) $\mathrm{mm}$ before IBEF and $8.4 \pm 3.8$ (range 3.6-13.3) $\mathrm{mm}$ after IBEF. According to the rule " $1 \mathrm{~mA}$ corresponds to $1 \mathrm{~mm}$," the localization of the CST as visualized by MEP-based tractography correlated with DSCS in 2 cases before the application of IBEF and in all cases after the application of IBEF. ${ }^{21,24}$ Table 4 gives detailed information on stimulation intensities and distances.

\section{Discussion}

\section{Clinical Relevance of IBEF}

The results of the present study show that the fusion of pre- and intraoperative MRI scans by IBEF is of clinical relevance. Through adjustment of the CST, we were able to show that the corrected subcortical course of the CST

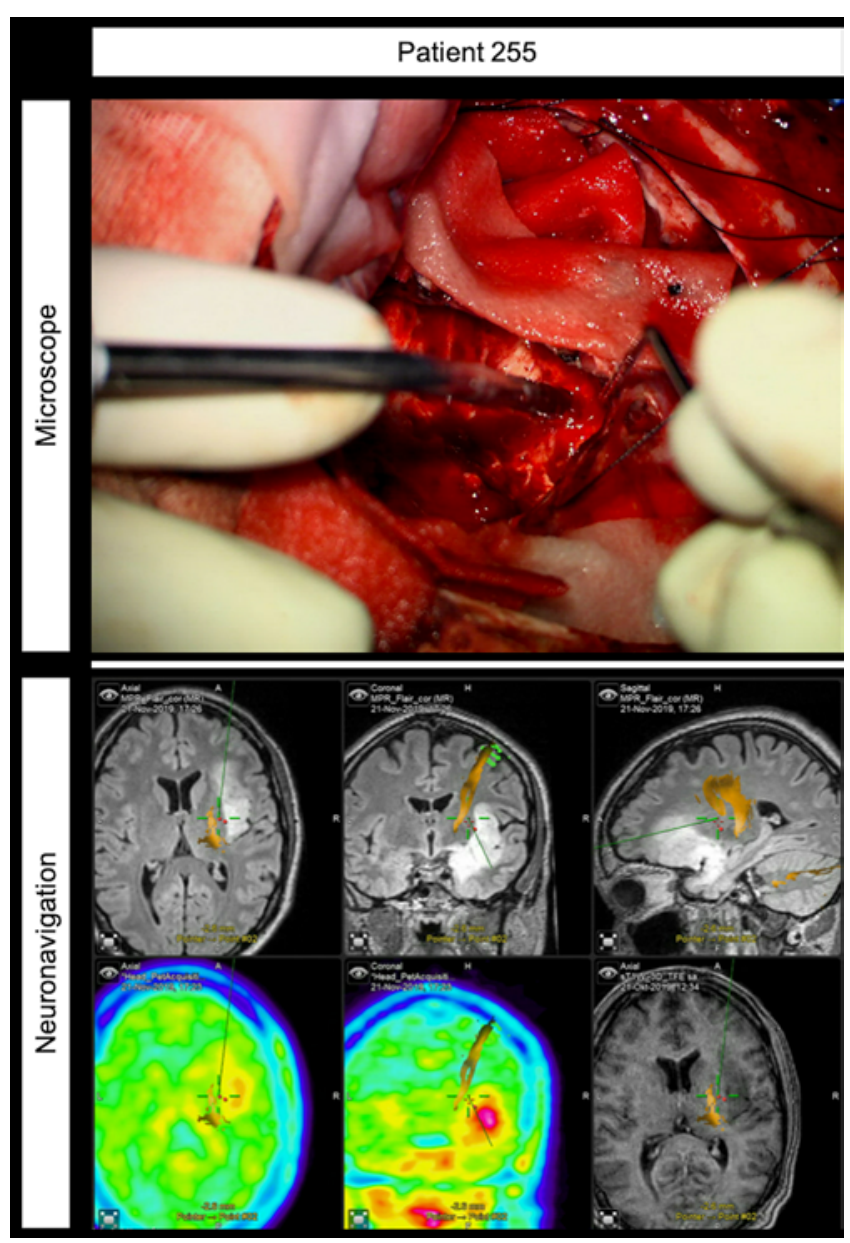

FIG. 2. Saving of subcortical stimulation points by neuronavigation. The figure demonstrates the 3D landmark of DSCS points (top) by neuronavigation (bottom) considering the example of patient 255. DSCS landmarks were saved at the location of the lowest current intensity of DSCS which still elicited an MEP response. Through neuronavigation, distances between DSCS points and the location of the CST could be measured as shown in Fig. 3.

correlated reliably to clinical outcome and IONM; without IBEF correction, this was not the case (all patients vs 3 patients; $p=0.0256$ ). Since these patients showed a loss of MEP amplitudes during tumor resection, we can also confirm that the correction of the CST by IBEF correlates with the results of IONM and the clinical long-term outcome. Additionally, we verified the location of the CST by DSCS in 4 cases. Thus, the obvious major inaccuracies of fusing preoperative tractography with ioMRI data due to missing 3D deformation can easily be addressed by IBEF.

Several studies have already confirmed that postoperative ischemic lesions as detected by early postoperative MRI correlate with new functional deficits after tumor resection. ${ }^{37-39}$ Moreover, IONM is an established technique that is standard for resection of eloquent brain tumors. . $^{5-9}$ Hence, with IONM and a lesion on MRI, we correlated the results of CST correction by IBEF to highly standardized techniques and found complete concordance in all cases. 
Ille et al.

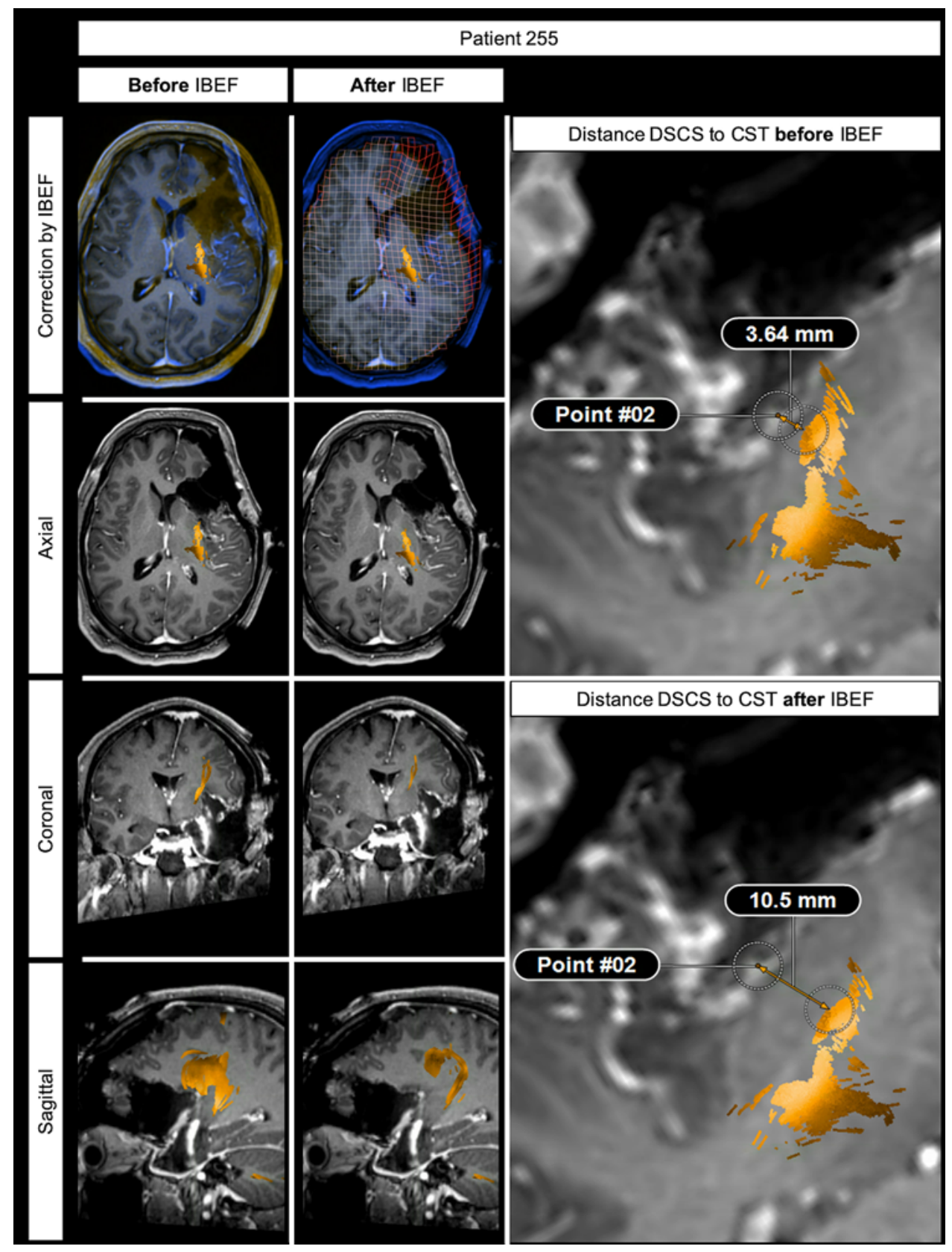

FIG. 3. Verification of CST visualization by DSCS. The figure shows the correction of the CST before (left column) and after (right column) IBEF within the ioMRI T1 + CA in axial, coronal, and sagittal slices as well as the measurement of distances between DSCS points (Point \#02) and the CST considering the example of patient 255. The top row shows the fusion (left) of preoperative (yellow) and intraoperative (blue) MRI scans and the extent of deformation (right) as indicated by red areas.

Despite our small sample size, these results are very clear. In our study, the CST was located within the lesioned region after IBEF in all patients. Inadvertently, all patients suffered from permanent surgery-related motor deficits.

\section{Visualization of the CST by Tractography}

The visualization of subcortical pathways by tractography is still an issue, although this technique has been repeatedly compared to IONM as the gold standard, with the two methods showing good correlations. ${ }^{10-15}$ Mainly, surgeons question the intraoperative use of tractography because of its inaccuracy after partial resection and consecutive brain shift, which is even aggravated toward the end of surgery when the resection gets close to highly eloquent tracts. However, during these last steps of resection, a reliable and accurate tractography method would be most useful.

This problem can now be solved using IBEF. It enables one to correct for brain shift and leads to a reliable tractography as shown by the present study's results, even if the 
TABLE 4. Comparison of DSCS and visualization of the CST

\begin{tabular}{|c|c|c|c|}
\hline \multirow[b]{2}{*}{ Patient No. } & \multirow{2}{*}{$\begin{array}{l}\text { DSCS Intensity } \\
(\mathrm{mA})\end{array}$} & \multicolumn{2}{|c|}{ Distance $(\mathrm{mm})$} \\
\hline & & Before IBEF & After IBEF \\
\hline 229 & 5 & 4.2 & 3.6 \\
\hline 255 & 12 & 3.6 & 10.5 \\
\hline 273 & 13 & 13.4 & 13.3 \\
\hline 298 & 5 & 14.5 & 6.1 \\
\hline
\end{tabular}

Lowest current intensities of DSCS that still elicited an MEP response and the distances between DSCS points and the CST as visualized by MEP-based tractography before and after the application of IBEF.

ioMRI is unable to perform intraoperative DTI sequences. Additionally, we were able to verify the location of the CST by DSCS.

The use of MEP-based tractography combining neurophysiologically acquired seed regions for DTI allows for a highly standardized visualization of the CST, ${ }^{33}$ and it has even been proven to correlate with clinical outcome in a considerably large cohort. ${ }^{40}$ Even more, we have now shown by DSCS verification that the technique of MEPbased tractography visualizes the correct location of the CST reliably if adapted by IBEF.

The present study results also confirm the clinical relevance of tractography and its ability to properly visualize the subcortical course of the CST. After correction of the CST after tumor resection, the location correlated with IONM results, DSCS, and the patients' long-term outcome in all cases. Thereby, IBEF qualifies for its application during tumor resections. In the case of tumor residuals, as shown by the ioMRI scan, the correction of preoperative tractography might contribute to the decision to perform further resection with the aim of preserving functionality, especially in combination with subcortical stimulation.

\section{Study Limitations}

Due to the rarity of the enrolled patients, the cohort size of the present study must be seen as the major limitation. However, despite the small sample size, the results are very clear and reach statistical significance due to the severe effect of the investigated intervention. We included only 8 patients with a loss of MEPs during the resection of motor-eloquent gliomas in order to definitively evaluate the accuracy and clinical relevance of IBEF with unalterable criteria. Additionally, we performed DSCS in 4 patients (including 1 of the original 8 patients) in order to directly provide proof for the correct spatial CST reconstruction by another modality. Within this cohort of patients, the results are highly homogenous and therefore reliable.

The data correlating CST reconstruction before and after IBEF with DSCS were less reliable because our ground truth observation was an apparent motor deficit, which is the most reliable measure.

\section{Conclusions}

IBEF proved to provide reliable adjustments of preoperatively acquired tractography and seems to fully correct for brain shift after ioMRI. Thus, IBEF should be implemented into the standard workflow as long as larger series do not prove the contrary, which is unlikely considering the clear data from the present study.

\section{Acknowledgments}

This trial was funded entirely by institutional grants from the Department of Neurosurgery, Technical University of Munich, School of Medicine, Klinikum rechts der Isar, Munich, Germany.

\section{References}

1. Stummer W, Reulen HJ, Meinel T, et al. Extent of resection and survival in glioblastoma multiforme: identification of and adjustment for bias. Neurosurgery. 2008;62(3):564-576.

2. Chang EF, Clark A, Smith JS, et al. Functional mappingguided resection of low-grade gliomas in eloquent areas of the brain: improvement of long-term survival. Clinical article. J Neurosurg. 2011;114(3):566-573.

3. Capelle L, Fontaine D, Mandonnet E, et al. Spontaneous and therapeutic prognostic factors in adult hemispheric World Health Organization Grade II gliomas: a series of 1097 cases: clinical article. J Neurosurg. 2013;118(6):1157-1168.

4. Sanai N, Polley MY, McDermott MW, et al. An extent of resection threshold for newly diagnosed glioblastomas. $J$ Neurosurg. 2011;115(1):3-8.

5. Deletis V. Intraoperative monitoring of the functional integrity of the motor pathways. Adv Neurol. 1993;63:201214.

6. De Witt Hamer PC, Robles SG, Zwinderman AH, et al. Impact of intraoperative stimulation brain mapping on glioma surgery outcome: a meta-analysis. J Clin Oncol. 2012; 30(20):2559-2565.

7. Southwell DG, Birk HS, Han SJ, et al. Resection of gliomas deemed inoperable by neurosurgeons based on preoperative imaging studies. J Neurosurg. 2018;129(3):567-575.

8. Sanai N, Berger MS. Mapping the horizon: techniques to optimize tumor resection before and during surgery. Clin Neurosurg. 2008;55:14-19.

9. Hervey-Jumper SL, Li J, Lau D, et al. Awake craniotomy to maximize glioma resection: methods and technical nuances over a 27-year period. J Neurosurg. 2015;123(2):325-339.

10. Henry RG, Berman JI, Nagarajan SS, et al. Subcortical pathways serving cortical language sites: initial experience with diffusion tensor imaging fiber tracking combined with intraoperative language mapping. Neuroimage. 2004;21(2): 616-622.

11. Leclercq D, Duffau H, Delmaire C, et al. Comparison of diffusion tensor imaging tractography of language tracts and intraoperative subcortical stimulations. J Neurosurg. 2010; 112(3):503-511.

12. Bello L, Castellano A, Fava E, et al. Intraoperative use of diffusion tensor imaging fiber tractography and subcortical mapping for resection of gliomas: technical considerations. Neurosurg Focus. 2010;28(2):E6.

13. Bello L, Gambini A, Castellano A, et al. Motor and language DTI fiber tracking combined with intraoperative subcortical mapping for surgical removal of gliomas. Neuroimage. 2008; 39(1):369-382.

14. Berman JI, Berger MS, Chung SW, et al. Accuracy of diffusion tensor magnetic resonance imaging tractography assessed using intraoperative subcortical stimulation mapping and magnetic source imaging. J Neurosurg. 2007;107(3): 488-494.

15. Berman JI, Berger MS, Mukherjee P, Henry RG. Diffusiontensor imaging-guided tracking of fibers of the pyramidal tract combined with intraoperative cortical stimulation mapping in patients with gliomas. J Neurosurg. 2004;101(1):66-72. 
16. Kamada K, Todo T, Ota T, et al. The motor-evoked potential threshold evaluated by tractography and electrical stimulation. J Neurosurg. 2009;111(4):785-795.

17. Kamada K, Todo T, Masutani Y, et al. Combined use of tractography-integrated functional neuronavigation and direct fiber stimulation. J Neurosurg. 2005;102(4):664-672.

18. Kinoshita M, Yamada K, Hashimoto N, et al. Fiber-tracking does not accurately estimate size of fiber bundle in pathological condition: initial neurosurgical experience using neuronavigation and subcortical white matter stimulation. Neuroimage. 2005;25(2):424-429.

19. Maesawa S, Fujii M, Nakahara N, et al. Intraoperative tractography and motor evoked potential (MEP) monitoring in surgery for gliomas around the corticospinal tract. World Neurosurg. 2010;74(1):153-161.

20. Mikuni N, Okada T, Nishida N, et al. Comparison between motor evoked potential recording and fiber tracking for estimating pyramidal tracts near brain tumors. J Neurosurg. 2007;106(1):128-133.

21. Nossek E, Korn A, Shahar T, et al. Intraoperative mapping and monitoring of the corticospinal tracts with neurophysiological assessment and 3-dimensional ultrasonographybased navigation. Clinical article. J Neurosurg. 2011;114(3): 738-746.

22. Prabhu SS, Gasco J, Tummala S, et al. Intraoperative magnetic resonance imaging-guided tractography with integrated monopolar subcortical functional mapping for resection of brain tumors. Clinical article. J Neurosurg. 2011;114(3):719-726.

23. Yamaguchi F, Takahashi H, Teramoto A. Navigation-assisted subcortical mapping: intraoperative motor tract detection by bipolar needle electrode in combination with neuronavigation system. J Neurooncol. 2009;93(1):121-125.

24. Schucht P, Seidel K, Jilch A, et al. A review of monopolar motor mapping and a comprehensive guide to continuous dynamic motor mapping for resection of motor eloquent brain tumors. Neurochirurgie. 2017;63(3):175-180.

25. Kubben PL, ter Meulen KJ, Schijns OE, et al. Intraoperative MRI-guided resection of glioblastoma multiforme: a systematic review. Lancet Oncol. 2011;12(11):1062-1070.

26. Li P, Qian R, Niu C, Fu X. Impact of intraoperative MRIguided resection on resection and survival in patient with gliomas: a meta-analysis. Curr Med Res Opin. 2017;33(4): 621-630.

27. Jenkinson MD, Barone DG, Bryant A, et al. Intraoperative imaging technology to maximise extent of resection for glioma. Cochrane Database Syst Rev. 2018;1:CD012788.

28. Cornaz F, Neidert MC, Piccirelli M, et al. Compatibility of intraoperative 3T MR imaging and intraoperative neurophysiological monitoring. Clin Neurophysiol. 2015;126(1): 218-220.

29. Sarnthein J, Lüchinger R, Piccirelli M, et al. Prevalence of complications in intraoperative magnetic resonance imaging combined with neurophysiologic monitoring. World Neurosurg. 2016;93:168-174.

30. Szelényi A, Gasser T, Seifert V. Intraoperative neurophysiological monitoring in an open low-field magnetic resonance imaging system: clinical experience and technical considerations. Neurosurgery. 2008;63(4)(suppl 2):268-276.

31. Breitkopf M, Bisdas S, Liebsch M, et al. Safety, utility, and clinical results of continuous intraoperative electrophysiologic monitoring in 1.5T iMRI-guided surgery. World Neurosurg. 2017;106:198-205.

32. Gerhardt J, Sollmann N, Hiepe P, et al. Retrospective distortion correction of diffusion tensor imaging data by semielastic image fusion-evaluation by means of anatomical landmarks. Clin Neurol Neurosurg. 2019;183:105387.

33. Krieg SM, Buchmann NH, Gempt J, et al. Diffusion tensor imaging fiber tracking using navigated brain stimulation-a feasibility study. Acta Neurochir (Wien). 2012;154(3):555-563.
34. Negwer C, Ille S, Hauck T, et al. Visualization of subcortical language pathways by diffusion tensor imaging fiber tracking based on rTMS language mapping. Brain Imaging Behav. 2017;11(3):899-914.

35. Dinevski N, Sarnthein J, Vasella F, et al. Postoperative neurosurgical infection rates after shared-resource intraoperative magnetic resonance imaging: a single-center experience with 195 cases. World Neurosurg. 2017;103:275-282.

36. Bloch O, Han SJ, Cha S, et al. Impact of extent of resection for recurrent glioblastoma on overall survival: clinical article. J Neurosurg. 2012;117(6):1032-1038.

37. Gempt J, Krieg SM, Hüttinger S, et al. Postoperative ischemic changes after glioma resection identified by diffusionweighted magnetic resonance imaging and their association with intraoperative motor evoked potentials. J Neurosurg. 2013;119(4):829-836.

38. Gempt J, Förschler A, Buchmann N, et al. Postoperative ischemic changes following resection of newly diagnosed and recurrent gliomas and their clinical relevance. J Neurosurg. 2013;118(4):801-808.

39. Jakola AS, Berntsen EM, Christensen P, et al. Surgically acquired deficits and diffusion weighted MRI changes after glioma resection-a matched case-control study with blinded neuroradiological assessment. PLoS One. 2014;9(7):e101805.

40. Sollmann N, Wildschuetz N, Kelm A, et al. Associations between clinical outcome and navigated transcranial magnetic stimulation characteristics in patients with motor-eloquent brain lesions: a combined navigated transcranial magnetic stimulation-diffusion tensor imaging fiber tracking approach J Neurosurg. 2018;128(3):800-810.

\section{Disclosures}

Dr. Meyer reports receiving honoraria, consulting fees, and research grants from Medtronic, Icotec AG, and Relievant Medsystems, Inc.; honoraria and research grants from Ulrich Medical; honoraria and consulting fees from Spineart Deutschland $\mathrm{GmbH}$ and DePuy Synthes; and royalties from Spineart Deutschland $\mathrm{GmbH}$. Dr. Krieg reports being a consultant for Nexstim Plc and Spineart Deutschland $\mathrm{GmbH}$ and receiving honoraria from Medtronic and Carl Zeiss Meditec. Drs. Krieg and Meyer report receiving research grants from and being consultants for Brainlab AG. Dr. Ille reports being a consultant for Brainlab AG. Dr. Kreiser reports being a consultant for Phenox and receiving honoraria from Philips, Penumbra, and Stryker.

\section{Author Contributions}

Conception and design: Krieg, Ille. Acquisition of data: Krieg, Ille, Schroeder, Wagner, Kreiser. Analysis and interpretation of data: Ille. Drafting the article: Ille. Critically revising the article: Krieg, Meyer. Reviewed submitted version of manuscript: Krieg, Schroeder, Wagner, Negwer, Kreiser, Meyer. Approved the final version of the manuscript on behalf of all authors: Krieg. Statistical analysis: Ille. Administrative/technical/material support: Krieg, Meyer. Study supervision: Krieg, Meyer.

\section{Supplemental Information}

\section{Previous Presentations}

Portions of this work were presented as an oral presentation and in e-poster form at the 88th (virtual) AANS Annual Scientific Meeting, April 25-29, 2020, and at the (virtual) Annual Meeting of the German Society for Neurosurgery (DGNC), June 24, 2020.

\section{Correspondence}

Sandro M. Krieg: Technische Universität München, Germany. sandro.krieg@tum.de. 